\title{
Fast Discovery of Spectrum Opportunities in Cognitive Radio Networks
}

\author{
Hyoil Kim and Kang G. Shin \\ Real-Time Computing Laboratory \\ Department of Electrical Engineering and Computer Science \\ The University of Michigan, Ann Arbor, MI 48109-2121 \\ Email: \{hyoilkim,kgshin\} @eecs.umich.edu
}

\begin{abstract}
We address the problem of rapidly discovering spectrum opportunities for seamless service provisioning for secondary users (SUs) in cognitive radio networks (CRNs). Specifically, we propose an efficient sensing-sequence that incurs a small opportunity-discovery delay by considering (1) the probability that a spectrum band (or a channel) may be available at the time of sensing, (2) the duration of sensing on a channel, and (3) the channel capacity. We derive the optimal sensing-sequence for channels with homogeneous capacities, and a suboptimal sequence for channels with heterogeneous capacities for which the problem of finding the optimal sensing-sequence is shown to be NP-hard.

To support the proposed sensing-sequence, we also propose a channel-management strategy that optimally selects and updates the list of backup channels. A hybrid of maximum likelihood (ML) and Bayesian inference is also introduced for flexible estimation of ON/OFF channel-usage patterns and prediction of channel availability when sensing produces infrequent samples.

The proposed schemes are evaluated via in-depth simulation. For the scenarios we considered, the proposed suboptimal sequence is shown to achieve close-to-optimal performance, reducing the opportunity-discovery delay by up to $47 \%$ over an existing probability-based sequence. The hybrid estimation strategy is also shown to outperform the ML-only strategy by reducing the overall opportunity-discovery delay by up to $34 \%$.
\end{abstract}

\section{INTRODUCTION}

Dynamic Spectrum Access (DSA) is key to solve the problem of wireless spectrum scarcity, rooted from inefficient spectrum utilization by the current static resource allocation policy. DSA can enhance spectrum utilization by allowing cognitive radios (CRs), also referred to as secondary users (SUs), to reuse legacy spectrum dynamically and opportunistically without interrupting incumbent/licensed spectrum users, also referred to as primary users (PUs).

Spectrum sensing plays an important role in realizing DSA, by (1) detecting PUs' presence, and (2) discovering spectrum opportunities. A spectrum sensor monitors a spectrum band/channel ${ }^{1}$ to detect PU signals and thus determine the channel's availability for use by SUs. If thus-discovered channels are utilized by SUs, they are referred to as in-band channels; else, they are called out-of-band channels.

Opportunity discovery is an act of searching for spectrum opportunities by sensing out-of-band channels. Opportunity

${ }^{1}$ Spectrum band and channel will be used interchangeably throughout this paper. (a) Event-driven channel reuse model

opportunity discovery channel reuse

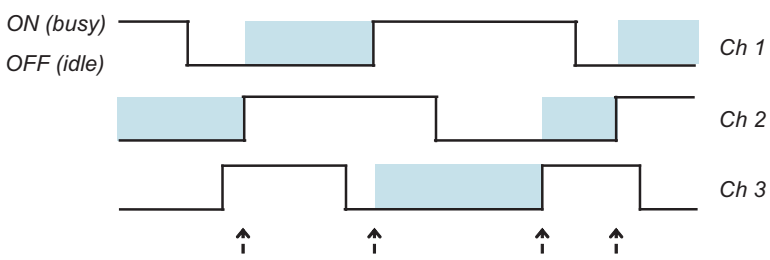

(b) Time-driven channel reuse model

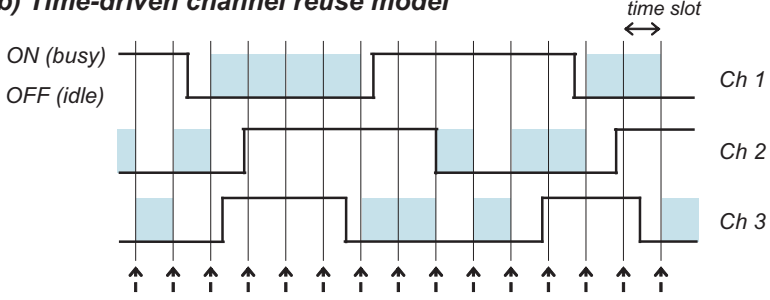

Fig. 1. Illustration of two channel reuse models, in case a CRN seeks a single idle channel at most. In general, however, a CRN may need more than one idle channel for its operation.

discovery is triggered when a CR network (CRN) experiences a shortage of opportunities to utilize, and it can be triggered periodically (time-driven) or on-demand (even-driven). In case of event-driven triggering, an in-band channel is utilized until its PUs' return to the channel which is detected by periodic in-band sensing. Upon the PUs' return, the channel must be vacated promptly. Opportunity discovery is triggered when a channel vacation makes the amount of opportunities (e.g., the total bandwidth of in-band channels) to be utilized drop below the spectrum demand of a CRN. In case of time-driven triggering, time is divided into slots, and an opportunity discovery is triggered at every slot. In-band channels discovered via opportunity discovery are utilized until the slot expires and hence, all in-band channels should be vacated at the end of the slot. The former model was introduced in [1], [2] and employed in IEEE 802.22 [3]. The latter model was used in [4]-[7]. Two channel reuse models are illustrated in Fig. 1.

\section{A. Motivation}

In both spectrum reuse models, fast opportunity discovery is essential to seamless service provisioning for CR devices. 
In CRNs, quality-of-service (QoS) depends greatly on the amount of explored spectrum opportunities since it determines the bandwidth provided to SUs and thus limits the maximum throughput in the CRN. The amount of explored opportunities, however, fluctuates in time due to frequent in-band channel vacations. Therefore, spectrum opportunities must be discovered as promptly and as much as needed at the time of each channel vacation, to avoid severe degradation of service quality.

To facilitate fast opportunity discovery, out-of-band channels are divided further into backup and candidate channels, as suggested in IEEE 802.22. Opportunity discovery searches backup channels for additional opportunities, where backup channels are specially chosen to maximize the chance of finding opportunities as much as needed. Out-of-band channels other than backup channels are referred to as candidate channels. Candidate channels are not searched until they are "imported" to the backup channel list. The list of backup (or candidate) channels is denoted as BCL (or CCL).

In this paper, we focus on fast discovery of spectrum opportunities via efficient scheduling of out-of-band spectrum sensing. Two important issues in opportunity discovery are considered: (1) how to build a sensing-sequence that helps find spectrum opportunities with minimal delay, and (2) how to construct and update BCL by importing/exporting channels from/to CCL.

\section{B. Contributions}

We propose a mechanism for fast opportunity discovery that consists of three parts to which our work makes main contributions. First, an optimal sensing-sequence is proposed to minimize the latency in finding the target amount of opportunities in BCL. We consider heterogeneous channel characteristics, including signal detection time $T_{I}^{i}$, channel capacity $C_{i}$, and the probability $P_{i d l e}^{i}$ of a channel to be idle. $^{2}$ The optimal sequence is derived for channels with homogeneous capacities, i.e., $C_{i}=C, \forall i$. For a more general case (i.e., channels with heterogeneous capacities), a necessary condition for optimality is derived. It is also shown that finding the optimal sequence is NP-hard, and hence, we propose a suboptimal sensing-sequence algorithm of polynomial time complexity. The efficiency of the algorithm is evaluated via simulation.

Second, optimal construction and efficient update of BCL is proposed. Our scheme suggests an optimal choice of initial backup channels that maximizes the chance of having enough opportunities in BCL while minimizing the size of BCL. An efficient BCL update algorithm is also proposed that imports/exports channels from/to CCL with less computational overhead so that BCL can be kept up-to-date.

Third, we propose a strategy that estimates ON/OFF channel-usage patterns and predicts channel availability by selectively applying maximum likelihood (ML) and/or Bayesian estimation. We capture the tradeoff between two estimation techniques: the former is simple but its performance degrades

\footnotetext{
${ }^{2}$ Note that we use $i$ as channel index in this paper.
}

greatly with infrequent samples; the latter requires more computation but performs better with a small number of samples [8] and is useful to model time-varying channel parameters by updating prior and posterior distributions. On the other hand, our scheme considers the impact of imperfect sensing on the prediction of channel availability $\left(P_{i d l e}^{i}\right)$, by considering probability of miss detection (PMD) and probability of false alarms (PFA).

\section{Related Work}

Among a number of studies on spectrum sensing, a few notable body of work is found to be related to fast opportunity discovery. Datla et al. [9] proposed a linear backoff, linearly decreasing the preference on sensing a channel whenever the channel is sensed 'occupied.' This is heuristic and does not optimize the opportunity-discovery delay. Zhao et al. [6] proposed a decentralized CR MAC protocol that senses a subset of backup channels at each time slot to jointly optimize sensing and transmission. However, the scheme does not prioritize the chosen channels, but rather senses all of them. Chang and Liu [4] suggested a strategy that optimally determines which channel to probe and when to transmit, but they focused on the case of single channel transmission only. Kim and Shin [2] introduced a sensing-sequence that sorts channels in descending order of the probability $P_{i d l e}^{i}$. However, such a sequence only maximizes the chance of finding an idle channel, instead of minimizing the overall discovery-delay. In IEEE 802.22 [10], the concept of backup and candidate channels are introduced to facilitate discovery of opportunities, but no algorithm is specified on how to construct BCL and CCL efficiently. Moreover, none of the above fully considered the heterogeneity of licensed channels.

On the other hand, Motamedi and Bahai [7] used Bayesian learning to predict the availability of a channel, where the learning process is simplified by assuming a geometric distribution for channel-usage patterns. In this paper, we use a general alternating renewal process and develop a multi-stage iterative Bayesian inference.

\section{Organization}

Section II briefly introduces basic assumptions and system models used in this paper. In Section III, we propose an efficient sensing-sequence that minimizes the opportunitydiscovery delay, by considering the heterogeneous characteristics of backup channels. Section IV presents construction of the initial BCL and an BCL-update algorithm to keep the list up-to-date. Section V introduces a strategy to estimate ON/OFF channel-usage patterns and predict channel availability during each opportunity discovery. The performance of the proposed schemes are evaluated in Section VI, and then the paper concludes in Section VII.

\section{System Model}

\section{A. Network Model}

A single-hop CRN with a group of SUs is considered, and the CRN is assumed to search $M$ licensed channels 

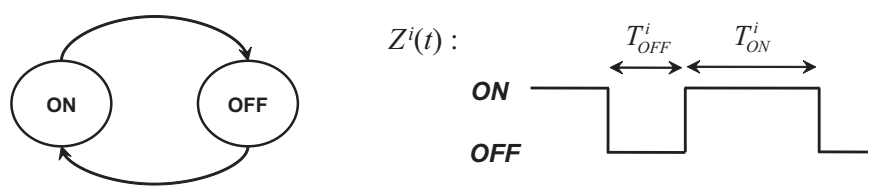

Fig. 2. Channel model: alternating renewal process with $\mathrm{ON}$ and OFF states

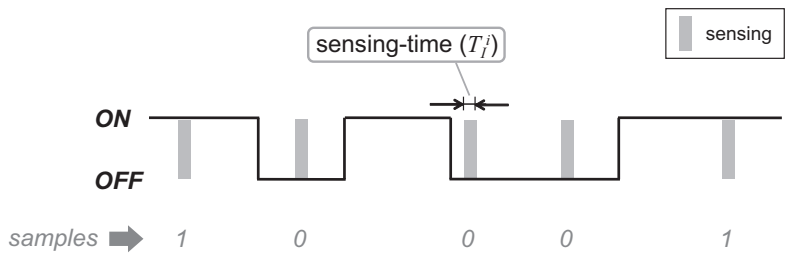

Fig. 3. Illustration of the sensing process on a channel

for spectrum opportunities it needs. Although our proposed schemes can also be applied to multi-hop CRNs, sensing in such a case must consider location-dependency of the observed signals. One possible approach is to divide the network into clusters, where SUs in each cluster cooperate for spectrum sensing and channel allocation. It is also assumed that there is no interference from other CRNs in $M$ channels, which can be accomplished by coordinated channel allocation between CRNs [1], [11].

Each SU is assumed to have been equipped with a single antenna, widely-tunable to any combination of $N$ channels by using signal processing techniques, such as NC-OFDM [12]. Having one antenna per SU may help reduce the size of a secondary device and avoid potential interference between colocated antennas due to their close proximity [13].

\section{B. Channel Model}

A channel is modeled as a renewal process alternating between $\mathrm{ON}$ and OFF states. The ON (OFF) state represents a time period within which a PU signal is present (absent). Once sensing finds a channel in its OFF state (i.e., an idle channel), SUs can utilize the channel until its next state transition to ON state. This type of channel model was introduced in [2], [7], [14] where its potential for modeling spectrum opportunities was demonstrated.

Fig. 2 illustrates the channel model. Suppose $i$ is the channel index $(i=1,2, \ldots, M)$, and let $Z^{i}(t)$ denote the state (ON or OFF) of channel $i$ at time $t$, such that

$$
\left\{\begin{array}{l}
Z^{i}(t)=1, \text { if channel } i \text { is ON (or busy) at } t, \\
Z^{i}(t)=0, \text { otherwise. }
\end{array}\right.
$$

For an alternating renewal channel [15], the sojourn times of $\mathrm{ON}$ and OFF states are represented by random variables $T_{O N}^{i}$ and $T_{O F F}^{i}$ with probability density functions (pdfs) $f_{T_{O N}^{i}}(t)$ and $f_{T_{O F F}^{i}}(t), t>0$, respectively. ${ }^{3} \mathrm{ON}$ and OFF states are also assumed to be independent of each other.

On the other hand, channel utilization, $u^{i} \in[0,1]$, defined as the average fraction of time during which channel $i$ is in

$$
{ }^{3} f_{T_{O N}^{i}}(t) \text { and } f_{T_{O F F}^{i}}(t) \text { can be any distribution functions. }
$$

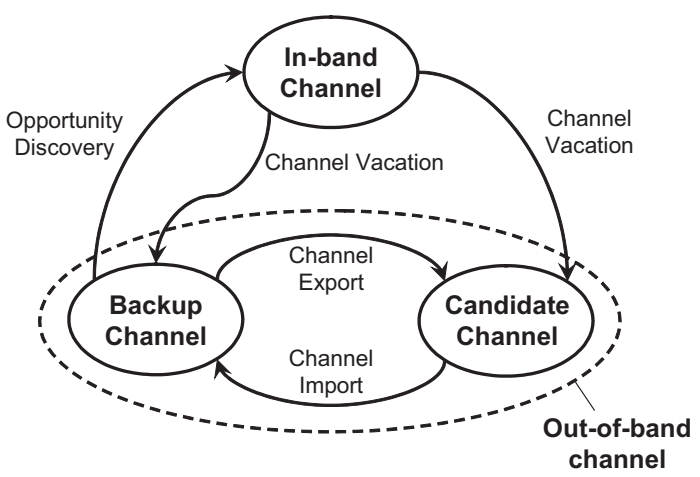

Fig. 4. The state transition diagram of a channel

ON state, is given as

$$
u^{i}=\frac{E\left[T_{O N}^{i}\right]}{E\left[T_{O N}^{i}\right]+E\left[T_{O F F}^{i}\right]} .
$$

\section{Sensing and Access Model}

A spectrum sensor is assumed to be co-located with a transceiver, i.e., a SU can be dynamically reconfigured to a transceiver or a sensor. When a SU acts as a spectrum sensor, it monitors channel $i$ during a certain time period, called sensingtime $T_{I}^{i}$, which is determined by the underlying detection method (e.g., energy or feature detection) and the type of PU signals [16]. $T_{I}^{i}$ is assumed small relative to $E\left[T_{O F F}^{i}\right]$ and $E\left[T_{O N}^{i}\right]$ such that channel $i$ 's state remains unchanged during the sensing-time. Sensing is akin to a sampling process, producing a binary random sequence since ON and OFF states correspond to sample 1 (busy) and 0 (idle), respectively. Fig. 3 illustrates this sensing process.

A CRN is assumed to require as much spectrum opportunities as $B_{r e q}$, which is the total spectrum demand from its SUs. Since all $C_{i}$ 's are not the same and could be smaller than $B_{\text {req }}$, $B_{\text {req }}$ may be fulfilled by finding more than one idle channel. Whenever $B_{r e q}$ is not met by the current in-band channels, an opportunity discovery is triggered and backup channels are searched sequentially during which SUs synchronously tune to one backup channel at a time following a given sensingsequence. Once a backup channel is detected idle, it becomes an in-band channel and is merged into one "logical" channel (i.e., a combination of all in-band channels) for SUs to utilize them. Opportunity discovery completes when the sum of inband channels' capacities reaches $B_{\text {req }}$.

The benefit of sequential search of backup channels is twofold: (1) it prevents a CRN from being partitioned while switching channels, and (2) it enhances the detectability of incumbents via collaborative sensing [17]-[19]. On the other hand, a CRN may choose a proper channel access mechanism (e.g., FDMA, TDMA, or CSMA) to determine how to reuse the logical channel, the choice of which is outside of the scope of this paper.

\section{Transition of Channel Association}

Fig. 4 illustrates the state transitions of a channel among three channel associations: in-band, backup, and candidate 
channels, where a state transition is triggered by one of the following four events: opportunity discovery, channel vacation, channel export, and channel import. First, during the opportunity discovery, a backup channel becomes an in-band channel if it is sensed idle. Next, in-band channel vacation makes the channel a backup or candidate channel, depending the channel's likelihood of having opportunities again in the near future. For example, an in-band channel with long ON/OFF periods (e.g., TV bands) is better put into CCL than BCL upon the channel's vacation since the channel would have been for a long ON period. On the other hand, channel exports/imports are triggered to update the entries of BCL: if a backup channel is less useful than a certain candidate channel, it may be exported to CCL, and the candidate channel can be imported to BCL instead. Further details on these state transitions will be presented in Section IV.

\section{Optimal Sensing Sequence for Fast Discovery OF OPPORTUNITIES}

In this section, we propose an efficient sensing-sequence of backup channels that incurs a small delay in discovering as much opportunities as a CRN needs. In building such a sequence, the heterogeneous characteristics of backup channels are considered by using a tuple of $\left\{T_{I}^{i}, C_{i}, P_{\text {idle }}^{i}\right\}$. $T_{I}^{i}$ may differ between channels because it depends on the type of PU signals. $C_{i}$ can be a physical bandwidth or Shannon capacity which varies with the time-varying channel condition (e.g., fading) and interference temperature [20]. $P_{\text {idle }}^{i}$ depends on the channel's ON/OFF usage pattern and hence varies with channels. Derivation of $P_{i d l e}^{i}$ for alternating renewal channels was introduced in [2].

Suppose there are $N(<M)$ backup channels with their $\left\{T_{I}^{i}, C_{i}, P_{i d l e}^{i}\right\}$ known, and $B_{r e q}$ is the amount of opportunities required for a CRN to support spectrum demands from its SUs. Then, upon triggering an opportunity discovery, the CRN needs to discover as much opportunities as $B=B_{\text {req }}-B_{\text {in-band }}$ where $B_{\text {in-band }}$ is the sum of in-band channels' capacities at the time of opportunity discovery. Note that $B_{i n-b a n d}=0$ in the time-driven channel reuse model.

\section{A. Optimal Sensing-Sequence: Analysis}

Let $S=\left\{s_{1}, s_{2}, \ldots, s_{N}\right\} \in \mathcal{S}$ be an ordered list of $N$ channels, where $s_{j}$ is the channel index of $j$-th channel in the sequence (i.e., $s_{j}$ : positive integer, $1 \leq s_{j} \leq N$ ) and $\mathcal{S}$ is the set of all possible channel sequences $\left(|\mathcal{S}|=N\right.$ !). Suppose $T_{I}^{i}$, $C_{i}$ and $P_{\text {idle }}^{i}=\operatorname{Pr}\left(\Theta_{i}=0\right)$ are known a priori, where $\Theta_{i} \in$ $\{0,1\}$ is the binary state of channel $i, i \in\{1,2, \ldots, N\}$ (' 0 ' means the channel is idle). Our objective is to determine the optimal sensing-sequence $S^{*}$ that minimizes the average delay in finding idle channels whose cumulative capacity exceeds $B$. This can be stated formally as

$$
\begin{aligned}
\text { Find } & S^{*}=\underset{S \in \mathcal{S}}{\operatorname{argmin}} E_{\tau}\left[\sum_{i=s_{1}}^{s_{\tau}} T_{I}^{i}\right] \\
\text { Subject to } & \sum_{i=s_{1}}^{s_{\tau-1}} C_{i} \cdot I_{\Theta_{i}}<B, \text { and } \sum_{i=s_{1}}^{s_{\tau}} C_{i} \cdot I_{\Theta_{i}} \geq B,
\end{aligned}
$$

where $I_{\Theta_{i}}$ is an indicator function such that

$$
I_{\Theta_{i}}=\left\{\begin{array}{l}
1, \text { if } \Theta_{i}=0, \\
0, \text { otherwise }
\end{array}\right.
$$

Note that $\tau$ is a random variable, and hence, the expected delay (i.e., average sensing-time) is considered in the objective function.

To find the optimal sequence, brute-force searching in $\mathcal{S}$ is not desirable since its computational complexity is $O(N !)$. Here, we derive an efficient channel-sorting algorithm to build an optimal sensing-sequence requiring much less computation. The proposed algorithm is based on the derivation of a necessary condition for optimality. We begin with introduction of some notations to use in the analysis.

Let $L$ be the optimal sensing-sequence and $L^{\prime}$ be its counterpart constructed by switching the order of $k$-th and $(k+1)$-th channels in $L$. That is,

$$
\begin{aligned}
L & =\left(l_{1}, \ldots, l_{k-1}, l_{k}, l_{k+1}, l_{k+2}, \ldots, l_{N}\right), \\
L^{\prime} & =\left(l_{1}, \ldots, l_{k-1}, l_{k+1}, l_{k}, l_{k+2}, \ldots, l_{N}\right) .
\end{aligned}
$$

On the other hand, $\mathcal{D}_{L}^{B}$ is defined as the average delay in locating idle channels whose cumulative capacity exceeds $B$, using a sensing-sequence $L . \mathcal{P}_{L}^{B}$ is defined as the probability that the sum of capacities of idle channels in a sensingsequence $L$ may be less than $B$. Formally, $\mathcal{D}_{L}^{B}$ and $\mathcal{P}_{L}^{B}$ are defined as follows:

$$
\begin{aligned}
& \mathcal{D}_{L}^{B}:=E_{\tau}\left[\sum_{i=l_{1}}^{l_{\tau}} T_{I}^{i}\right], \\
& \mathcal{P}_{L}^{B}:=\operatorname{Pr}\left(\sum_{i \in L} C_{i} \cdot I_{\Theta_{i}}<B\right) .
\end{aligned}
$$

In addition, let us define the following ordered lists:

$$
\begin{aligned}
L_{k-1} & =\left(l_{1}, l_{2}, \ldots, l_{k-1}\right), \\
L_{k} & =\left(l_{1}, l_{2}, \ldots, l_{k-1}, l_{k}\right), \\
L_{k-1, k+1} & =\left(l_{1}, l_{2}, \ldots, l_{k-1}, l_{k+1}\right), \\
L_{k+1} & =\left(l_{1}, l_{2}, \ldots, l_{k-1}, l_{k}, l_{k+1}\right), \\
L_{k-1, k+1, k} & =\left(l_{1}, l_{2}, \ldots, l_{k-1}, l_{k+1}, l_{k}\right), \\
L_{k+1}^{c} & =\left(l_{k+2}, \ldots, l_{N}\right) .
\end{aligned}
$$

Since a channel is sensed only when those channels preceding in the list provide less opportunities than $B$, we can express $\mathcal{D}_{L}^{B}$ and $\mathcal{D}_{L^{\prime}}^{B}$ as

$$
\begin{aligned}
\mathcal{D}_{L}^{B}= & \mathcal{D}_{L_{k-1}}^{B}+\mathcal{P}_{L_{k-1}}^{B} \cdot T_{I}^{l_{k}}+\mathcal{P}_{L_{k}}^{B} \cdot T_{I}^{l_{k+1}} \\
& +\mathcal{P}_{L_{k+1}}^{B} \cdot \mathcal{D}_{L_{k+1}^{c}}^{B}, \\
\mathcal{D}_{L^{\prime}}^{B}= & \mathcal{D}_{L_{k-1}}^{B}+\mathcal{P}_{L_{k-1}}^{B} \cdot T_{I}^{l_{k+1}}+\mathcal{P}_{L_{k-1, k+1}}^{B} \cdot T_{I}^{l_{k}} \\
& +\mathcal{P}_{L_{k-1, k+1, k}}^{B} \cdot \mathcal{D}_{L_{k+1}^{c}}^{B} .
\end{aligned}
$$

Since $\mathcal{D}_{L}^{B} \leq \mathcal{D}_{L^{\prime}}^{B}$ and $\mathcal{P}_{L_{k+1}}^{B}=\mathcal{P}_{L_{k-1, k+1, k}}^{B}$, we have

$$
\mathcal{P}_{L_{k-1}}^{B} \cdot T_{I}^{l_{k}}+\mathcal{P}_{L_{k}}^{B} \cdot T_{I}^{l_{k+1}} \leq \mathcal{P}_{L_{k-1}}^{B} \cdot T_{I}^{l_{k+1}}+\mathcal{P}_{L_{k-1, k+1}}^{B} \cdot T_{I}^{l_{k}}
$$


Using $\mathcal{P}_{L_{k}}^{B}=\mathcal{P}_{L_{k-1}}^{B} \cdot\left(1-P_{i d l e}^{l_{k}}\right)+\mathcal{P}_{L_{k-1}}^{B-C_{l_{k}}} \cdot P_{i d l e}^{l_{k}}$, the above equation reduces to:

$$
\frac{T_{I}^{l_{k}}}{\left(\mathcal{P}_{L_{k-1}}^{B}-\mathcal{P}_{L_{k-1}}^{B-C_{l_{k}}}\right) P_{i d l e}^{l_{k}}} \leq \frac{T_{I}^{l_{k+1}}}{\left(\mathcal{P}_{L_{k-1}}^{B}-\mathcal{P}_{L_{k-1}}^{B-C_{l_{k+1}}}\right) P_{\text {idle }}^{l_{k+1}}}
$$

\section{B. Optimal Sensing-Sequence for a Special Case: Homoge- neous Channel Capacities}

For homogeneous channel capacities (i.e., $C_{i}=C, \forall i$ ), the optimal sensing-sequence is determined as shown in Theorem 1 .

Theorem 1: If $C_{i}=C, \forall i$, then the optimal sensingsequence is built by sorting channels in ascending order of $T_{I}^{i} / P_{i d l e}^{i}$.

Proof: By substituting $C$ for $C_{l_{k}}$ and $C_{l_{k+1}}$ in Eq. (2), the inequality condition reduces to:

$$
\frac{T_{I}^{l_{k}}}{P_{i d l e}^{l_{k}}} \leq \frac{T_{I}^{l_{k+1}}}{P_{\text {idle }}^{l_{k+1}}}, \text { for } 1 \leq k \leq N-1,
$$

which is a necessary condition for optimality. However, since there exists a single and unique sequence satisfying such a necessary condition, ${ }^{4}$ the condition also becomes sufficient. Therefore, the resulting sequence is optimal.

\section{Suboptimal Sensing-Sequence for Heterogeneous Channels}

With a more general channel condition (i.e., heterogeneous channel capacities), however, there is no handy rule like Eq. (3). The form of Eq. (2) suggests that the problem of optimal channel ordering is state-dependent: the $k$-th channel in the optimal sequence is determined by considering $L_{k-1}$, which is a sub-sequence of $S^{*}$ with the first $k-1$ entries. To find the optimal sequence, all $N$ ! possible sequences must be searched to find a complete set of sequences satisfying Eq. (2) since there could be more than one such sequence. Once such sequences are found, their $\mathcal{D}_{L}^{B}$ must be compared to find the one providing minimal $\mathcal{D}_{L}^{B}$. Therefore, the problem of finding the optimal sequence again becomes as complex as brute-force search (i.e., $O(N !)$ ), which is NP-hard.

Here, we propose a suboptimal algorithm of polynomial time complexity while guaranteeing discovery of a sensing sequence that satisfies the optimality condition Eq. (2). In this algorithm, $S^{*}$ is formed by iteratively determining the $k$-th entry $k=1,2, \ldots, N$ while updating $L_{k-1}$. Let's denote by stage $k$ the $k$-th iteration of finding the $k$-th entry. At stage $k, T_{I}^{i} /\left\{\left(\mathcal{P}_{L_{k-1}}^{B}-\mathcal{P}_{L_{k-1}}^{B-C_{i}}\right) P_{i d l e}^{i}\right\}$ are calculated for $N-k+1$ channels (except $k-1$ channels in $L_{k-1}$ ). Then, the channel with minimal $T_{I}^{i} /\left\{\left(\mathcal{P}_{L_{k-1}}^{B}-\mathcal{P}_{L_{k-1}}^{B-C_{i}}\right) P_{\text {idle }}^{i}\right\}$ is picked as $k$ th entry of $S^{*}$. The computational complexity of the proposed algorithm is $O\left(N^{2}\right)$, since $N+(N-1)+\ldots+1=N(N+1) / 2$. In Section VI, it will be shown that the proposed suboptimal algorithm achieves near-optimal performance under the various channel conditions tested.

\footnotetext{
${ }^{4}$ if we deal with the sequences, without differentiating them, which are created by switching the order of channels with same $T_{I}^{i} / P_{i d l e}^{i}$.
}

The initial step (i.e., $k=1$ ) of the algorithm must be handled carefully, because $L_{k-1}=\emptyset$ and $\left(\mathcal{P}_{L_{k-1}}^{B}-\mathcal{P}_{L_{k-1}}^{B-C_{l_{k}}}\right)=0$ at $k=1$. Let $L$ and $L^{\prime}$ be defined the same as before except that they differ by the first two entries in the sequence. That is,

$$
\begin{aligned}
L & =\left(l_{1}, l_{2}, l_{3}, \ldots, l_{N}\right), \\
L^{\prime} & =\left(l_{2}, l_{1}, l_{3}, \ldots, l_{N}\right) .
\end{aligned}
$$

Then,

$$
\begin{aligned}
& \mathcal{D}_{L}^{B}=T_{I}^{l_{1}}+\mathcal{P}_{\left\{l_{1}\right\}}^{B} \cdot T_{I}^{l_{2}}+\mathcal{P}_{\left\{l_{1}, l_{2}\right\}}^{B} \cdot \mathcal{D}_{\left\{l_{3}, \ldots, l_{N}\right\}}^{B}, \\
& \mathcal{D}_{L^{\prime}}^{B}=T_{I}^{l_{2}}+\mathcal{P}_{\left\{l_{2}\right\}}^{B} \cdot T_{I}^{l_{1}}+\mathcal{P}_{\left\{l_{2}, l_{1}\right\}}^{B} \cdot \mathcal{D}_{\left\{l_{3}, \ldots, l_{N}\right\}}^{B},
\end{aligned}
$$

which gives

$$
\left(1-\mathcal{P}_{\left\{l_{2}\right\}}^{B}\right) \cdot T_{I}^{l_{1}} \leq\left(1-\mathcal{P}_{\left\{l_{1}\right\}}^{B}\right) \cdot T_{I}^{l_{2}} .
$$

Considering

$$
\mathcal{P}_{\left\{l_{k}\right\}}^{B}=\left\{\begin{array}{l}
1-P_{i d l e}^{l_{k}}, \text { if } C_{l_{k}} \geq B \\
1, \text { otherwise }
\end{array}\right.
$$

we can have the following three cases.

Case 1: $C_{l_{1}} \geq B$, and $C_{i}<B$ for $i \neq l_{1}$. In this case, Eq. (4) becomes

$$
0 \cdot T_{I}^{l_{1}} \leq P_{i d l e}^{l_{1}} \cdot T_{I}^{i}
$$

which is always true since $P_{i d l e}^{l_{1}} \cdot T_{I}^{i}>0$. Therefore, we schedule channel $l_{1}$ first.

Case 2: there exist at least two channels with $\boldsymbol{C}_{\boldsymbol{i}} \geq \boldsymbol{B}$. Let the indices of such channels be $l_{k}$ and $l_{k+1}$. Then, Eq. (4) becomes

$$
\frac{T_{I}^{l_{k}}}{P_{i d l e}^{l_{k}}} \leq \frac{T_{I}^{l_{k+1}}}{P_{i d l e}^{l_{k+1}}}
$$

Therefore, the first channel to be scheduled is the one having minimum $\frac{T_{I}^{i}}{P_{\text {idle }}^{i}}$ among those with $C_{i} \geq B$.

Case 3: $\boldsymbol{C}_{\boldsymbol{i}} \stackrel{\text { idle }}{<} \boldsymbol{B}, \forall \boldsymbol{i}$. In such a case, Eq. (4) becomes $0 \leq 0$. To avoid a random choice, we follow the special case rule in Theorem 1: the first channel is the one with minimum $\frac{T_{I}^{i}}{P_{i d l e}^{i}}$.

The proposed sensing-sequence algorithm is described in Fig. 5.

\section{Discussion}

A CRN may sometimes fail to find the necessary amount $B$ of opportunities after searching $N$ channels. In such a case, the CRN must retry opportunity discovery until enough opportunities are discovered. We set the retry period to tRETRY which is a design parameter.

Once the first opportunity discovery fails, the overall discovery delay until $B$ is accomplished depends more on $t R E T R Y$, rather than the optimality of sensing sequence. Therefore, it is desirable to have an enough number of 'good' channels in BCL so that opportunity discovery may be successful at the first trial. The construction of such BCL will be discussed in the next section. 


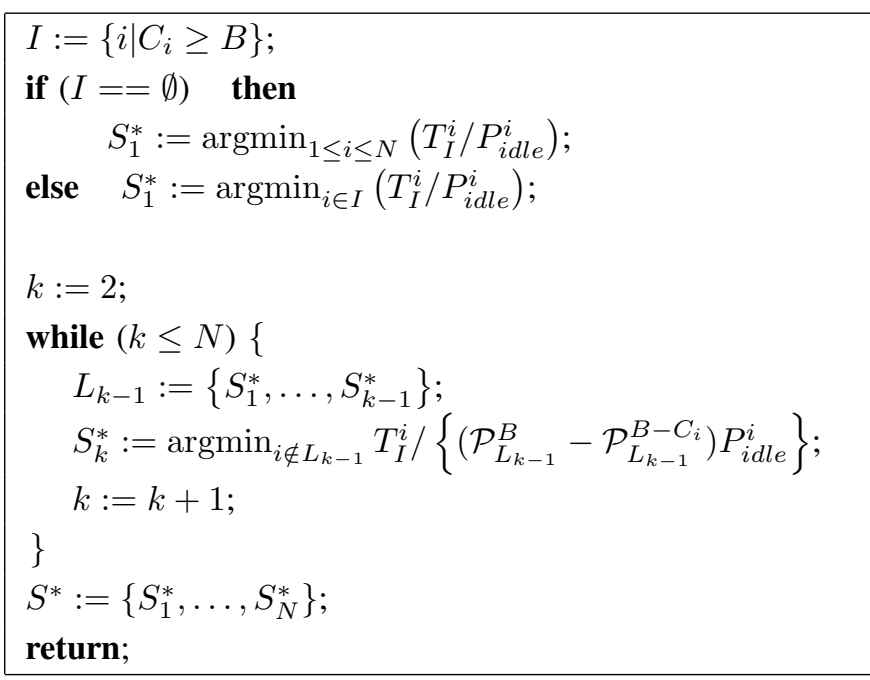

Fig. 5. Pseudo-code of the optimal sensing-sequence algorithm

\section{BaCkup Channel List (BCL) Management}

\section{A. Construction of Initial BCL}

When a BCL is constructed initially, there could be many candidates for its entries. In IEEE 802.22, for example, there are $68 \mathrm{TV}$ channels (channels 2 to 69) in the VHF/UHF bands (54-806 MHz) [21]. If CR devices are allowed to operate in heterogenous spectrum bands, the number of candidate channels may even grow larger.

Upon selecting the initial backup channels, two conflicting objectives must be met: the BCL should (1) contain as few channels as possible since the cost of channel sequencing grows fast at the rate $O\left(N^{2}\right)$ as shown in Section III, and (2) have many 'good' channels to increase the chance of finding enough opportunities at the first opportunity-discovery attempt. To achieve both objectives, we propose the following strategy: first, all $M$ licensed channels are ordered according to the sensing-sequence algorithm in Section III, and then the initial BCL is constructed by choosing the first $N$ channels of the sequence where $N$ is minimized while achieving the second objective.

The problem of constructing the initial BCL is formally stated as follows. Suppose $L_{M}=\left\{l_{1}^{*}, l_{2}^{*}, \ldots, l_{M}^{*}\right\}$ is the (sub)optimally-ordered list of $M$ channels. Also, let $L_{N}=$ $\left\{l_{1}^{*}, l_{2}^{*}, \ldots, l_{N}^{*}\right\}, N \leq M$ be a sub-list of $L_{M}$ with its first $N$ entries. Our objective is to find an optimal $N$ such that $N$ channels may contain opportunities more than $B_{r e q}$ with probability thPOTENTIAL, which is a pre-defined threshold (e.g., thPOTENTIAL $=0.9$ ). That is,

$$
\begin{aligned}
& N^{*}=\min \left\{N \mid \mathcal{C}_{L_{N}}^{B_{\text {req }}} \geq \text { thPOTENTIAL }\right\} \\
& \mathcal{C}_{L_{N}}^{B_{\text {req }}}:=\operatorname{Pr}\left\{\sum_{i \in L_{N}} C_{i} \cdot I_{\Theta_{i}} \geq B_{\text {req }}\right\}=1-\mathcal{P}_{L_{N}}^{B_{\text {req }}},
\end{aligned}
$$

where $\mathcal{C}_{L_{N}}^{B_{r e q}}$, capacity potential of $B_{\text {req }}$ in $L_{N}$, represents the probability that $L_{N}$ may contain more opportunities than $B_{\text {req }}$. We assume $\mathcal{C}_{L_{M}}^{B_{\text {req }}} \geq$ thPOTENTIAL.
Initially, $P_{i d l e}^{i}$ cannot be predicted correctly as there is no sample collected from any channel. Once a channel becomes a backup, it may be sensed during opportunity-discovery attempts (by sequential sensing of channels in BCL), and the collected samples are then used to estimate the channel's ON/OFF pattern. The resulting estimates will be used to predict $P_{\text {idle. }}^{i}{ }^{5}$

Nevertheless, it may be possible to obtain some prior knowledge on $P_{i d l e}^{i}$. For example, a certain TV channel's average broadcasting time is known to be 18 hours per day, so we can assume the initial $P_{\text {idle }}^{i}$ to be $1 / 4$. If there is no such information available, a best guess will be $P_{i d l e}^{i}=1 / 2$.

\section{B. BCL Update Strategy}

The initial entries of BCL may need to be updated since they were chosen by guessing $P_{i d l e}^{i}$. As samples are accumulated on backup channels, the optimal entries can be more accurately derived. If channels have time-invariant ON/OFF distributions $f_{T_{O N}^{i}}(t)$ and $f_{T_{O F F}^{i}}(t)$, the best BCL update strategy consists of:

- Learning: by extensively sensing $M$ channels, collect enough samples for each channel and produce accurate estimates.

- Single-time optimization: order $M$ channels and construct the BCL optimally. Keep this BCL forever.

Unfortunately, channels are usually time-varying, rendering the above strategy ineffective. Instead, the BCL could be reconstructed periodically by sorting all $M$ channels repeatedly. However, its large overhead of sorting $M$ channels makes the approach impractical. So, we propose an efficient and lightweight BCL update strategy that sorts BCL or CCL separately and only when necessary, with no sampling required on candidate channels. In this strategy, BCL is updated periodically every tUPDATE seconds. At every BCL update, $\mathcal{C}_{L_{N}}^{B_{\text {req }}}$ is calculated with most recent channel estimates, where $L_{N}$ is the current BCL with $N$ backup channels. According to $\mathcal{C}_{L_{N}}^{B_{\text {req }} \text {, }}$, one of the following actions is taken: channel export (BCL $\rightarrow$ $\mathrm{CCL}$ ), channel import $(\mathrm{BCL} \leftarrow \mathrm{CCL})$, channel swap (BCL $\leftrightarrow$ $\mathrm{CCL})$, and mandatory channel export $(\mathrm{BCL} \rightarrow \mathrm{CCL})$.

1) Channel export: If $\mathcal{C}_{L_{N}}^{B_{\text {req }}}>$ thPOTENTIAL $L^{\text {upper }}$, we export a certain number of least preferred channels from BCL since it contains more channels than necessary. We use thPOTENTIAL ${ }^{\text {upper }}=$ thPOTENTIAL $+\epsilon_{1}\left(\epsilon_{1}>0\right)$ to avoid any impetuous channel export. To export channels, the (sub)optimal sequence of all $N$ (not $M$ !) backup channels is constructed and the optimal BCL size $N^{*}$ is calculated again. Then, the last $\left(N-N^{*}\right)$ channels in the sequence are exported to CCL.

2) Channel import: If $\mathcal{C}_{L_{N}}^{B_{\text {req }}}<$ thPOTENTIAL $L_{\text {lower }}$, a number of candidate channels are imported from CCL to satisfy $\mathcal{C}_{L^{\prime}}^{B_{\text {req }}} \geq$ thPOTENTIAL, where $L_{N}^{\prime}$ is an extended BCL after importing the CCL channels. We use thPOTENTIAL $L_{\text {lower }}=$ thPOTENTIAL $-\epsilon_{2}\left(\epsilon_{2}>0\right)$ to

\footnotetext{
${ }^{5}$ Section $\mathrm{V}$ will cover more details on ON/OFF-pattern estimation and $P_{i d l e}^{i}$ prediction.
} 
avoid impetuous channel import. To import channels, candidate channels are sorted in the (sub)optimal order, and are imported to BCL one by one in the order of preference until $\mathcal{C}_{L^{\prime}}^{B_{\text {req }}} \geq$ thPOTENTIAL is met.

3) Channel swap: One may want to restrict the size of BCL within some range such as $N_{\text {lower }} \leq N^{*} \leq N^{\text {upper }}$. $N_{\text {lower }}$ helps reserve a minimal number of backup channels so that opportunity-discovery would be successful, and $N^{u p p e r}$ upperbounds the computational overhead in sorting backup channels. When $N_{\text {lower }}$ and $N^{u p p e r}$ are used, channel export (or import) cannot be processed if $N^{*}=N_{\text {lower }}$ (or $N^{u p p e r}$ ). In such a case, we swap the least preferred backup channel with the most preferred candidate channel if the swap helps decrease/increase $\mathcal{C}_{L_{N}}^{B_{r e q}}$ as desired.

4) Mandatory channel export: In our scheme, channels are categorized into two classes: (1) those with long ON/OFF periods (class- $L$ ), and (2) those with short ON/OFF periods (class-S). The former includes TV bands where ON/OFF periods are in the order of hours at least, and the latter includes 802.11 channels where ON/OFF periods typically last tens of milliseconds [7].

A mandatory channel export is triggered when a classL channel (either in-band or backup channel) is sampled to be 'ON' (i.e., busy). Such a class-L channel is better to be expelled from BCL since the channel is unlikely to become available soon. Once expelled, the channel is forced to stay in CCL until its $t N O_{-} I M P O R T \_T I M E R$ expires. $T N O \_I M P O R T \_T I M E R$ is a design parameter and can be uniquely determined for each channel. A similar concept was found in IEEE 802.22 [10], where a backup channel detected busy is marked as 'occupied by PUs' and never sensed until Non-Occupancy Period (recommended to be 10 minutes) expires. Note that candidate channels with their $t N O \_I M P O R T \_T I M E R$ unexpired are not considered for channel import.

\section{Channel State Prediction Strategy}

Prediction of channel availability (i.e., $P_{i d l e}^{i}$ ) at the time of opportunity discovery is indispensable to achieving the minimal discovery-delay since $P_{i d l e}^{i}$ is one of the key factors in the construction of a (sub)optimal sensing-sequence. For alternating renewal channels, Kim and Shin [2] derived $P_{i d l e}^{i}$ with a given set of samples while considering the correlation between the samples.

$P_{i d l e}^{i}$ is formulated by the estimates on ON/OFF channelusage patterns, and hence, the estimation method should be carefully chosen to produce accurate estimates. In addition, samples collected by the spectrum sensor does not always represent the actual channel state, because there is no perfect signal detector with $P M D=P F A=0$. Our proposed approach to the above two issues will be discussed in the following subsections.

\section{A. Estimation of Channel-Usage Patterns}

In [2] we introduced ML estimation for renewal channels and also showed that, when ML estimation is em- ployed, the sampling rate must be lower-bounded to achieve accurate estimates on channel-usage patterns. Specifically, the sampling period must be set to be proportional to $\min \left\{E\left[T_{O N}^{i}\right], E\left[T_{O F F}^{i}\right]\right\}$. Therefore, class-S channels must be sensed more frequently than class-L channels, to achieve the same level of accuracy. However, performing additional sensing on class-S channels may incur a high sensingoverhead.

To achieve accurate estimation on class-S channels without requiring additional sensing, Bayesian estimation can be used. Unlike large-sample asymptotic estimators (e.g., ML) whose estimation accuracy degrades as the channel is less infrequently sampled, Bayesian estimation is known to perform reasonably well even if the number/frequency of samples is limited [8]. Bayesian inference is also useful to model timevarying parameters by updating prior and posterior distributions of the unknown parameters.

Using these features of Bayesian estimation, we propose the following strategy for accurate estimation of channel-usage patterns.

- Class-L channels: perform ML estimation.

- Class-S channels: perform Bayesian estimation.

Although Bayesian learning on channel availability has been introduced in [7], it models a channel yielding uncorrelated samples and considers stationary probability $u^{i}$ for $P_{i d l e}^{i}$. With alternating renewal channels, however, samples are correlated, and hence, the model in [7] is not suitable. Therefore, we propose an iterative Bayesian inference for alternating renewal channels. A single-step Bayesian inference and its extension to a multi-stage iterative estimation will be used. We will then discuss how to reduce the computational complexity of Bayesian estimation.

1) Single-Step Bayesian Inference: A single-step Bayesian inference [8] is summarized as follows. Suppose a sequence of $k$ samples $\mathbf{Z}_{k}^{i}=\left(Z_{t_{1}}^{i}, Z_{t_{2}}^{i}, \ldots, Z_{t_{k}}^{i}\right)$ is given for channel $i$, where $t_{j}$ denotes a timestamp of the $j$-th sample. For an alternating renewal channel, the joint probability mass function (pmf) of $\mathbf{Z}_{k}^{i}$ is denoted as $f\left(\mathbf{Z}_{k}^{i} \mid \boldsymbol{\theta}^{i}\right)$, which depends on the vector $\boldsymbol{\theta}^{i} \in \boldsymbol{\Theta}^{i}$ of the distribution parameters of $f_{T_{O N}^{i}}(t)$ and $f_{T_{O F F}^{i}}(t)$ [2]. When $\pi\left(\boldsymbol{\theta}^{i}\right)$ is a prior (subjective) distribution of $\boldsymbol{\theta}^{i}$, the posterior distribution of $\boldsymbol{\theta}^{i}$ with a new observation $\mathbf{Z}_{k}^{i}$ is

$$
\pi\left(\boldsymbol{\theta}^{i} \mid \mathbf{Z}_{k}^{i}\right)=\frac{\pi\left(\boldsymbol{\theta}^{i}\right) f\left(\mathbf{Z}_{k}^{i} \mid \boldsymbol{\theta}^{i}\right)}{m\left(\mathbf{Z}_{k}^{i}\right)}=\frac{\pi\left(\boldsymbol{\theta}^{i}\right) f\left(\mathbf{Z}_{k}^{i} \mid \boldsymbol{\theta}^{i}\right)}{\int_{\Theta^{i}} \pi\left(\boldsymbol{\theta}^{i}\right) f\left(\mathbf{Z}_{k}^{i} \mid \boldsymbol{\theta}^{i}\right) d \boldsymbol{\theta}^{i}},
$$

where $m\left(\mathbf{Z}_{k}^{i}\right)$ is the marginal joint pmf of $\mathbf{Z}_{k}^{i}$. Then, the estimates of $\boldsymbol{\theta}^{i}$ are obtained as

$$
\hat{\boldsymbol{\theta}}^{i}=E^{\pi\left(\boldsymbol{\theta}^{i} \mid \mathbf{Z}_{k}^{i}\right)}\left[\boldsymbol{\theta}^{i}\right],
$$

where $E[\cdot]$ is taken over the posterior distribution $\pi\left(\boldsymbol{\theta}^{i} \mid \mathbf{Z}_{k}^{i}\right)$.

2) Iterative Bayesian Inference: We extend the single-step procedure in $\mathrm{V}-\mathrm{A} 1$ to provide an iterative Bayesian process where estimates are produced each time a new sample is collected. Fig. 6 illustrates the concept of our iterative Bayesian inference. The process starts with an initial prior distribution 


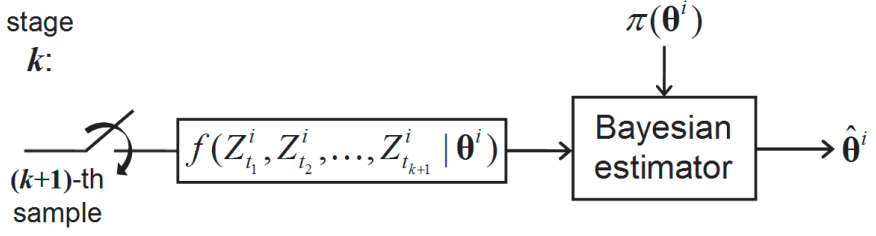

Fig. 6. Iterative Bayesian inference

$\pi\left(\boldsymbol{\theta}^{i}\right)$, and the first stage begins upon collection of the first two samples. Upon arrival of the $(k+1)$-th sample (i.e., at stage $k$ ), the $k$-th pair of new estimates are computed by using $\pi\left(\boldsymbol{\theta}^{i}\right)$ and $f\left(\mathbf{Z}_{k+1}^{i} \mid \boldsymbol{\theta}^{i}\right)$ of $(k+1)$ samples.

From now on, the channel index $i$ will be omitted when it does not cause any ambiguity, since the estimation procedure is independent for each channel.

As assumed in Section II-B, a channel is considered as an alternating renewal process with $\mathrm{ON}$ and OFF states. For an illustrative purpose, exponentially-distributed ON and OFF periods are considered with pdfs:

$$
\left\{\begin{array}{l}
f_{T_{O F F}}(t)=\theta_{O F F} e^{-\theta_{O F F} t}(t>0) \\
f_{T_{O N}}(t)=\theta_{O N} e^{-\theta_{O N} t}(t>0)
\end{array}\right.
$$

Therefore, unknown channel parameters are given as

$$
\boldsymbol{\theta}=\left(\theta_{O N}, \theta_{O F F}\right), \quad \boldsymbol{\Theta}=\{0, \infty\} \times\{0, \infty\} .
$$

It should be noted, however, that the proposed procedure can be applied to any general pdfs of $T_{O N}$ and $T_{O F F}$.

The initial prior distribution $\pi(\boldsymbol{\theta})$ is usually chosen with subjective reasoning. The criteria in selecting the prior is based on the prior knowledge of $\boldsymbol{\theta}$. For exponentially-distributed $\mathrm{ON}$ and OFF periods, $\pi(\boldsymbol{\theta})=\pi\left(\theta_{O N}, \theta_{O F F}\right)$ should be chosen to satisfy the following condition:

$$
\theta_{O N}>0, \theta_{O F F}>0,
$$

by the definition of exponential distribution. On the other hand, if some statistics are available on average ON and OFF periods on a large time-scale (e.g., a day or a week), such knowledge can be reflected in the choice of the prior. For example, suppose $\tau_{O N}$ and $\tau_{O F F}$ are the average $O N$ and OFF periods in a day. Then, the prior knowledge can be used to form $\pi(\boldsymbol{\theta})$ such that

$$
\tau_{O N}=1 / E\left[\theta_{O N}\right], \quad \tau_{O F F}=1 / E\left[\theta_{O F F}\right],
$$

since $\theta_{O N}=1 / E\left[T_{O N}\right]$ and $\theta_{O F F}=1 / E\left[T_{O F F}\right]$.

Here we assume $\tau_{O N}$ and $\tau_{O F F}$ are given, and the prior distribution is set as

$$
\pi\left(\theta_{O N}, \theta_{O F F}\right)=\tau_{O N} e^{-\tau_{O N} \theta_{O N}} \cdot \tau_{O F F} e^{-\tau_{O F F} \theta_{O F F}},
$$

or equivalently

$$
\left\{\begin{array}{l}
\pi\left(u, \theta_{O F F}\right)=\tau_{O N} \tau_{O F F} e^{\left(\tau_{O N}-\tau_{O F F}-\tau_{O N} / u\right) \theta_{O F F}}, \\
u=\theta_{O F F} /\left(\theta_{O N}+\theta_{O F F}\right)
\end{array}\right.
$$

where $\theta_{O N}$ and $\theta_{O F F}$ are assumed to be exponentiallydistributed with mean $\tau_{O N}$ and $\tau_{O F F}$, respectively. ${ }^{6}$ Setting the prior distribution as above can satisfy the conditions (5) and (6).

Considering the fact that an alternating renewal process is semi-Markov [15], $f\left(\mathbf{Z}_{k+1} \mid \boldsymbol{\theta}\right)$ at stage $k$ becomes

$$
f\left(\mathbf{Z}_{k+1} \mid \boldsymbol{\theta}\right)=f\left(Z_{t_{1}} \mid \boldsymbol{\theta}\right) f\left(Z_{t_{2}} \mid Z_{t_{1}}, \boldsymbol{\theta}\right) \cdots f\left(Z_{t_{k+1}} \mid Z_{t_{k}}, \boldsymbol{\theta}\right) .
$$

The derivation of the transition probability $f\left(Z_{t_{j+1}} \mid Z_{t_{j}}, \boldsymbol{\theta}\right)$, $j=1,2, \ldots, k$, for arbitrarily-formed $f_{T_{O N}}(t)$ and $f_{T_{O F F}}(t)$ can be found in [15]. For example, with exponentiallydistributed ON and OFF periods, we can show

$$
\begin{aligned}
f\left(Z_{t_{1}} \mid \boldsymbol{\theta}\right)= & (1-u)^{1-Z_{t_{1}}} u^{Z_{t_{1}}} \\
f\left(Z_{t_{j+1}} \mid Z_{t_{j}}, \boldsymbol{\theta}\right)= & (1-u)^{1-Z_{t_{j+1}}} \cdot u^{Z_{t_{j+1}}}+(-1)^{Z_{t_{j}}+Z_{t_{j+1}}} . \\
& u^{1-Z_{t_{j}}} \cdot(1-u)^{Z_{t_{j}}} \cdot e^{-\theta_{O F F} \Delta_{j} / u}
\end{aligned}
$$

where $\Delta_{j}=t_{j+1}-t_{j}$.

Now, $m_{k}\left(\mathbf{Z}_{k+1}\right)$ at stage $k$ is derived as

$$
\begin{aligned}
m_{k}\left(\mathbf{Z}_{k+1}\right)= & \int_{0}^{\infty} \int_{0}^{\infty} \pi(\boldsymbol{\theta}) f\left(\mathbf{Z}_{k+1} \mid \boldsymbol{\theta}\right) d \theta_{O N} d \theta_{O F F} \\
= & \int_{0}^{1} \int_{0}^{\infty} \pi\left(u, \theta_{O F F}\right) \cdot(1-u)^{1-Z_{t_{1}}} u^{Z_{t_{1}}} \\
& \cdot\left\{\prod_{j=1}^{k} f\left(Z_{t_{j+1}} \mid Z_{t_{j}}, \boldsymbol{\theta}\right)\right\} \cdot\left(\frac{\theta_{O F F}}{u^{2}}\right) d \theta_{O F F} d u,
\end{aligned}
$$

which provides a closed-form solution by transforming the product of sums with $k$ terms, $\prod_{j=1}^{k} f\left(Z_{t_{j+1}} \mid Z_{t_{j}}, \boldsymbol{\theta}\right)$, into a sum of products with $2^{k}$ terms. We then obtain two estimates $\hat{\theta}_{O N}$ and $\hat{\theta}_{O F F}$ at stage $k$ as

$$
\begin{aligned}
\hat{\theta}_{O N, k}= & \int_{0}^{\infty} \int_{0}^{\infty} \theta_{O N} \cdot \pi\left(\boldsymbol{\theta} \mid \mathbf{Z}_{k+1}\right) d \theta_{O N} d \theta_{O F F} \\
= & \int_{0}^{1} \int_{0}^{\infty} \theta_{O F F}\left(\frac{1}{u}-1\right) \frac{\pi\left(u, \theta_{O F F}\right) f\left(\mathbf{Z}_{k+1} \mid \boldsymbol{\theta}\right)}{m_{k}\left(\mathbf{Z}_{k+1}\right)} \\
& \cdot\left(\frac{\theta_{O F F}}{u^{2}}\right) d \theta_{O F F} d u
\end{aligned}
$$

and

$$
\begin{aligned}
\hat{\theta}_{O F F, k}= & \int_{0}^{\infty} \int_{0}^{\infty} \theta_{O F F} \cdot \pi\left(\boldsymbol{\theta} \mid \mathbf{Z}_{k+1}\right) d \theta_{O N} d \theta_{O F F} \\
= & \int_{0}^{1} \int_{0}^{\infty} \theta_{O F F} \frac{\pi\left(u, \theta_{O F F}\right) f\left(\mathbf{Z}_{k+1} \mid \boldsymbol{\theta}\right)}{m_{k}\left(\mathbf{Z}_{k+1}\right)} \\
& \cdot\left(\frac{\theta_{O F F}}{u^{2}}\right) d \theta_{O F F} d u
\end{aligned}
$$

both of which provide closed-form estimators with the same transformation as in $m_{k}\left(\mathbf{Z}_{k+1}\right)$. The derived Bayesian estimators work as fast as ML estimators since both are expressed in closed forms.

\footnotetext{
${ }^{6}$ Note that modeling $\theta_{O F F}$ and $\theta_{O N}$ to be exponentially-distributed has nothing to do with exponentially-distributed ON and OFF periods.
} 
3) Implementation Issues: Despite its simplicity and good performance, Bayesian inference suffers from computational complexity inherent in integration of pdfs to produce $m_{k}\left(\mathbf{Z}_{k+1}\right)$ and $\boldsymbol{\theta}$. In case there are no closed-form solutions for them, the complexity of $h\left(\mathbf{Z}_{k+1}, \boldsymbol{\theta}\right)$ grows exponentially as the number of stages increases, although the number of samples increases linearly.

To overcome this problem, a few adjustments can be made to the proposed scheme. First, MAX_BS_STAGE, a design parameter, can be set so that the process resets to stage 1 whenever the current stage number reaches $M A X \_B S \_S T A G E$. When it resets, the prior distribution $\pi(\boldsymbol{\theta})$ is updated with the most recent estimates. That is, $\tau_{O N}$ and $\tau_{O F F}$ in $\pi(\boldsymbol{\theta})$ must be replaced by $1 / \hat{\theta}_{O N}$ and $1 / \hat{\theta}_{O F F}$ where $\hat{\theta}_{O N}$ and $\hat{\theta}_{O F F}$ are the most recent estimates.

Next, a pre-computed look-up table can also be used to evaluate the integrals. When an integration does not provide an analytical solution, numerical integration (e.g., Simpson's rule) or Monte Carlo integration [8] can be used. Through a series of computations, the estimates of unknown parameters can be pre-computed with sample values and their timestamps as input arguments. This way, the delay involved with computational complexity of Bayesian estimation can be bounded reasonably small.

\section{B. Compensation of Signal Detection Error}

Using ML or Bayesian estimation, the unknown distribution parameters of ON/OFF periods can be computed. The next step is then to accurately predict $P_{i d l e}^{i}$ using the estimates.

As discussed in Section I-B, the effect of imperfect sensing (i.e., $P M D, P F A>0$ ) should be accounted for to make $P_{\text {idle }}^{i}$ reflect the actual channel state. Here we present a Bayesian state estimation procedure that can compensate for the sensing error. It is shown in [2] that with alternating renewal channels, $P_{i d l e}^{i}$ becomes the transition probability between samples, which is often nonlinear. In such a case, Bayesian state estimation is a proper choice because it is a nonlinear estimation technique, while other linear estimators (e.g., extended Kalman filter (EKF)) just approximate nonlinear estimation [22].

The procedure of Bayesian state estimation is summarized as follows [22]. Suppose $X_{t_{k}} \in\{0,1\}$ denotes the actual state of a channel at time $t_{k}$, and $Z_{t_{k}} \in\{0,1\}$ denotes the observed channel state (i.e., a sample) at time $t_{k}$. Also, suppose the state-transition function $g_{k}$ and measurement function $l_{k}$ are nonlinear and time-varying such that

$$
X_{t_{k+1}}=g_{k}\left(X_{t_{k}}\right) \text {, and } Z_{t_{k}}=l_{k}\left(X_{t_{k}}, w_{k}\right),
$$

where $w_{k}$ is the measurement noise. Assuming the pdf of the initial state $X_{t_{0}}$ is known, the estimator is initialized as

$$
f\left(X_{t_{0}} \mid \mathbf{Z}_{0}\right)=f\left(X_{t_{0}}\right)
$$

where $\mathbf{Z}_{k}=\left\{Z_{t_{1}}, Z_{t_{2}}, \ldots, Z_{t_{k}}\right\}$ for $k \geq 1$ and $\mathbf{Z}_{0}=\emptyset$. For each $k \geq 1$, we evaluate

$$
\begin{aligned}
f\left(X_{t_{k}} \mid \mathbf{Z}_{k-1}\right) & =\sum_{X_{t_{k-1}}} f\left(X_{t_{k}} \mid X_{t_{k-1}}\right) f\left(X_{t_{k-1}} \mid \mathbf{Z}_{k-1}\right), \\
f\left(X_{t_{k}} \mid \mathbf{Z}_{k}\right) & =\frac{f\left(Z_{t_{k}} \mid X_{t_{k}}\right) f\left(X_{t_{k}} \mid \mathbf{Z}_{k-1}\right)}{\sum_{X_{t_{k}}} f\left(Z_{t_{k}} \mid X_{t_{k}}\right) f\left(X_{t_{k}} \mid \mathbf{Z}_{k-1}\right)},
\end{aligned}
$$

where $f\left(X_{t_{k}} \mid \mathbf{Z}_{k-1}\right)$ is the prior pmf of $X_{t_{k}}$ before observing $Z_{t_{k}}$ and $f\left(X_{t_{k}} \mid \mathbf{Z}_{k}\right)$ is the posterior pmf of $X_{t_{k}}$ after observing $Z_{t_{k}}$.

Prior and posterior pmfs are updated whenever a new sample $Z_{t_{k}}$ is obtained. Since channel parameters $u$ and $\theta_{O F F}$ are time-varying, updating prior and posterior pmfs must be preceded by the Bayesian inference on them (Section V-A). Then, when an opportunity-discovery is triggered at time $t$, $P_{\text {idle }}(t)$ can be estimated as

$$
P_{\text {idle }}(t)=\left.f\left(X_{t_{k}} \mid \mathbf{Z}_{k-1}\right)\right|_{t_{k}=t, X_{t_{k}}=0} .
$$

In the above procedure, $f\left(X_{t_{k}} \mid X_{t_{k-1}}\right)$ and $f\left(Z_{t_{k}} \mid X_{t_{k}}\right)$ are yet to be determined. For exponentially-distributed $\mathrm{ON}$ and OFF periods, $f\left(X_{t_{k}} \mid X_{t_{k-1}}\right)$ is given as in Eq. (7). On the other hand, by definition of $P_{D}$ and $P_{F}$, it is clear that

$$
f\left(Z_{t_{k}} \mid X_{t_{k}}\right)=\left\{\begin{array}{lc}
1-P_{F}, & \text { if }\left(X_{t_{k}}, Z_{t_{k}}\right)=(0,0), \\
P_{F}, & \text { if }\left(X_{t_{k}}, Z_{t_{k}}\right)=(0,1), \\
1-P_{D}, & \text { if }\left(X_{t_{k}}, Z_{t_{k}}\right)=(1,0), \\
P_{D}, & \text { if }\left(X_{t_{k}}, Z_{t_{k}}\right)=(1,1) .
\end{array}\right.
$$

\section{Vi. Performance Evaluation}

To demonstrate the efficacy of the proposed schemes, we conducted four types of simulation. The first test in Section VI-A compares the average opportunity-discovery delay of the proposed sensing sequence with the optimal delay (computed using a brute-force search). This test will show that the proposed suboptimal algorithm performs reasonably well and sometimes performs as good as the optimal sensingsequence. The second test in Section VI-B shows the superiority of the proposed sensing sequence to the probabilitybased sensing-sequence in [2]. The third test in Section VI-C demonstrates the performance improvement of the BCL update strategy by comparing the proposed scheme with the case of no BCL update. The last test in Section VI-D evaluates the benefit of selecting between ML and Bayesian by comparing its performance with ML-only estimation. The simulation parameters for those tests are presented in the corresponding subsections.

For all tests, we use the average delay as a yardstick in discovering opportunities. The average discovery-delay is captured by considering two different cases: (1) when opportunity discovery completes during the first round of searching backup channels, and (2) when it completes during the successive retries, provided the first round failed. The delay in the first case says how efficient a sensing sequence is, whereas the second case shows how efficiently the BCL is constructed/updated so that opportunity discovery may be successful at early rounds. 


\begin{tabular}{|l|l||l|l||l|l|}
\hline \multicolumn{2}{|c|}{ Test 1a: varying $\overline{\mathbf{C}}$ (fixing $s_{\mathbf{C}}^{2}=1.08$ ) } & \multicolumn{2}{|c||}{ Test 1b: varying $\overline{\mathbf{T}}_{I}$ (fixing $s_{\mathbf{T}_{I}}^{2}=12.96$ ) } & \multicolumn{2}{l|}{ Test 1c: varying $\overline{\mathbf{u}}$ (fixing $s_{\mathbf{u}}^{2}=0.216$ ) } \\
\hline$u^{i}$ & $0.15+0.1(i-1)$ & $u^{i}$ & $0.15+0.1(i-1)$ & $u^{i}$ & $(\overline{\mathbf{u}}-0.3)+0.1(i-1)$ \\
\hline$T_{I}^{i}$ & $48-6(i-1)$ & $T_{I}^{i}$ & $\left(\overline{\mathbf{T}}_{I}+18\right)-6(i-1)$ & $T_{I}^{i}$ & $48-6(i-1)$ \\
\hline$C_{i}$ & $(\overline{\mathbf{C}}-1.5)+0.5(i-1)$ & $C_{i}$ & $1+0.5(i-1)$ & $C_{i}$ & $1+0.5(i-1)$ \\
\hline$\overline{\mathbf{C}}$ & from 2.0 to 4.5 in step of 0.25 & $\overline{\mathbf{T}}_{I}$ & from 25 to 35 in step of 1 & $\overline{\mathbf{u}}$ & from 0.35 to 0.55 in step of 0.02 \\
\hline
\end{tabular}

TABLE I

Channel Parameters for TeSt $1: T_{I}^{i}$ IN $m s e c, 1 \leq i \leq N=7$

\begin{tabular}{|c|l|}
\hline$u^{i}$ & $0.14+0.04(i-1)$ \\
\hline$T_{I}^{i}$ & $50(i=1,2,3), 40(i=4,5,6), 30(i=7,8)$, \\
$(\mathrm{msec})$ & $20(i=9,10,11), 10(i=12,13,14,15)$ \\
\hline$C_{i}$ & $0.5+0.5(i-1)$ \\
\hline$E\left[T_{O N}^{i}\right]$ & $i \leq 8: 0.25+0.25(i-1), i \geq 9: 8.5+0.25(i-9)$ \\
\hline
\end{tabular}

TABLE II

ChANNEl PARAMETERS FOR TEST $2: 1 \leq i \leq N=15$

Channels are simulated as alternating renewal processes with exponentially-distributed $\mathrm{ON}$ and OFF periods. The channel parameters $\theta_{O N}$ and $\theta_{O F F}$ are assumed time-varying and increasing/decreasing by $10 \%$ every 100 seconds. To track the time-varying channel condition, a moving window of $t M O V I N G_{-}$WINDOW $=20$ seconds is used for each channel with which previously collected samples older than tMOVING_WINDOW are discarded. We adopt the event-driven channel reuse model for simulations. That is, once a backup channel is sensed idle during an opportunity discovery, it becomes an in-band channel and reused until it switches to ON state.

It is assumed that sensing can accurately measure the actual channel state (i.e., $P M D \approx 0$ and $P F A \approx 0$ ). Although $P M D>0$ and $P F A>0$ in reality, this assumption will help us focus on the efficacy of the proposed sensing-sequence and BCL update strategies. It should be noted, however, that this assumption is made only for an illustrative purpose, and our schemes can adopt the scheme in Section V-B to reflect imperfect sensing.

For every test, a single simulation ran for 3,000 seconds, and the same test was repeated 10 times to take the average performance. In addition, $t R E T R Y$ is set to 0.1 .

\section{A. Test 1: Effectiveness of the Proposed (Sub)optimal Sensing- Sequence}

In this test, the average discovery-delay of the proposed (sub)optimal sensing-sequence is compared with the minimum, median, maximum delays found by trying all $N$ ! possible sequences via a brute-force search. Assuming perfect knowledge on channel parameters, the average delay is analytically derived by using the stationary probability $u^{i}$ such as $P_{\text {idle }}^{i}=1-u^{i}{ }^{7}$ The derivation excludes the case when there are less than $B_{r e q}$ opportunities in $N$ channels. We fix $N=7$ and BCL is not updated so as to focus on the analytically achievable performance of the sequences.

Suppose $\mathbf{u}=\left(u^{1}, u^{2}, \ldots, u^{N}\right), \mathbf{T}_{I}=\left(T_{I}^{1}, T_{I}^{2}, \ldots, T_{I}^{N}\right)$, and $\mathbf{C}=\left(C_{1}, C_{2}, \ldots, C_{N}\right)$. Then, for $B_{r e q}=5$, various

\footnotetext{
${ }^{7}$ Using $1-u^{i}$ for $P_{\text {idle }}^{i}$ is to obtain an analytical average delay. In other tests, $P_{i d l e}^{i}$ is derived using transition probabilities.
}

\begin{tabular}{|c|l|}
\hline$u^{i}$ & $0.225+0.025(i-1)$ \\
\hline$T_{I}^{i}$ & $50(1 \leq i \leq 4), 40(5 \leq i \leq 8), 30(9 \leq i \leq 12)$ \\
$(\mathrm{msec})$ & $20(13 \leq i \leq 16), 10(17 \leq i \leq 20)$ \\
\hline$C_{i}$ & $0.75+0.35(i-1)$ \\
\hline$E\left[T_{O N}^{i}\right]$ & $i \leq 12$ (class-S): $0.20+0.10(i-1)$ \\
& $i \geq 13$ (class-L): $10.25+0.25(i-13)$ \\
\hline
\end{tabular}

TABLE III

Channel Parameters For Tests 3 And $4: 1 \leq i \leq N=20$

combinations of channel conditions are considered with a tuple of $\left\{\mathbf{u}, \mathbf{T}_{I}, \mathbf{C}\right\}$ by (1) varying $\overline{\mathbf{C}}$ while fixing $s_{\mathbf{C}}^{2}$, where $\overline{\mathbf{C}}$ and $s_{\mathbf{C}}^{2}$ are sample mean and sample variance of $\mathbf{C}$, respectively, ${ }^{8}$ (2) varying $\overline{\mathbf{T}}_{I}$ while fixing $s_{\mathbf{T}_{I}}^{2}$, and (3) varying $\overline{\mathbf{u}}$ while fixing $s_{\mathbf{u}}^{2}$. The simulation parameters are listed in Table I.

Fig. 7 plots the simulation results. Interestingly, the proposed sequence is achieving the optimal performance in Test 1a, regardless of the average channel capacity. However, in Test $1 \mathrm{~b}$ and $1 \mathrm{c}$, there are certain thresholds of the average sensing-time and channel-utilization, above which performance degrades. In other words, the proposed sequence performs exceptionally well unless we have long sensingtimes or highly-utilized channels. Considering the fact that CR targets under-utilized channels for its application, sensing-time seems more important in this phenomenon. We are currently investigating how to enhance the proposed suboptimal algorithm by addressing the above issue, which is part of our future work.

\section{B. Test 2: Performance Enhancement of the Proposed Sensing- Sequence against Other Schemes}

In this test, the proposed sensing-sequence is compared with a probability-based sequence in [2]. Channel conditions are chosen such that they can reveal the inapplicability of the probability-based scheme. For example, channels with low utilization may be preferred by the scheme in [2], although it is better to put low priority on such channels if they have small capacity and long sensing-times. Assuming the perfect knowledge ${ }^{9}$ on channel conditions, both schemes are simulated with $B_{r e q}=8,10$, and 12 . The number of channels is fixed at 15 and no BCL update is performed. The parameters used for this simulation are listed in Table II.

In Fig. 8, 'Random' represents the sequence with randomlysorted channels and 'Worst' means the one built by reversing the proposed sequence. On the other hand, Delay Type-I is the average delay when opportunity discovery is successful

\footnotetext{
${ }^{8}$ Specifically, $\overline{\mathbf{C}}=\frac{1}{N} \sum_{i=1}^{N} C_{i}$ and $s_{\mathbf{C}}^{2}=\frac{1}{N-1} \sum_{i=1}^{N}\left(C_{i}-\overline{\mathbf{C}}\right)^{2}$.

${ }^{9}$ The impact of estimation will be investigated in the next two tests.
} 

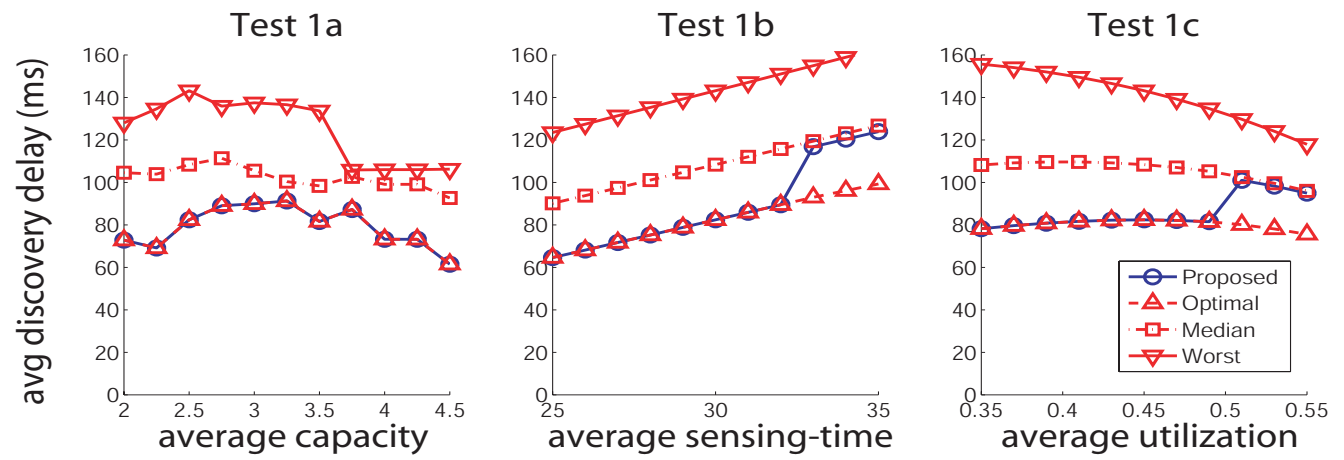

Fig. 7. Test $1-\mathrm{a} / \mathrm{b} / \mathrm{c}$ : average delay of the proposed suboptimal sequence vs. optimal delay

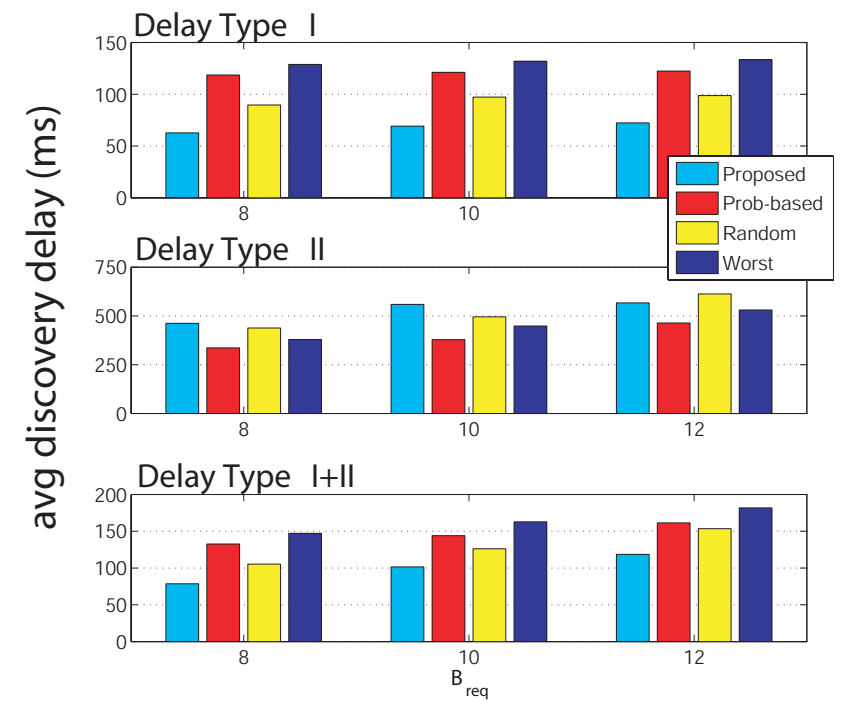

Fig. 8. Test 2: proposed sensing-sequence vs. probability-based sequence

without any retry, whereas Delay Type-II is the average delay when the first trial fails and thus opportunity discovery completes after successive retries (by tRETRY). Delay Type$I+I I$ is the overall average delay considering both cases.

The proposed scheme is shown to enhance Type-I delay by up to $47.12 \%$ and $30.07 \%$ over 'Prob-based' and 'Random', respectively. The overall delay (i.e., Type-I+II) is also enhanced by up to $40.70 \%$ and $25.28 \%$, respectively. It can also be seen that the proposed sequence does not reduce TypeII delay in this case, which is expected because the channel set is fixed and no BCL update is performed for this test. It will become clear in Test 3 that BCL update can reduce Type-II delay significantly by refreshing backup channels with more promising ones, that maximizes the chance of completing opportunity discovery without retries.

\section{Test 3: Proposed BCL Update Strategy vs. No BCL Update}

In this test, the efficiency of the proposed BCL update strategy is evaluated and compared with another scheme with no BCL update. Both schemes initialize BCL by optimally determining the size of $\mathrm{BCL}$ and its initial entries, with $P_{i d l e}^{i}=1 / 2, \forall i$ assuming no prior knowledge on the ON/OFF usage patterns. As the simulation progresses, the proposed scheme updates BCL via channel import/export/swap and adjusts the BCL size accordingly, whereas the latter scheme al-
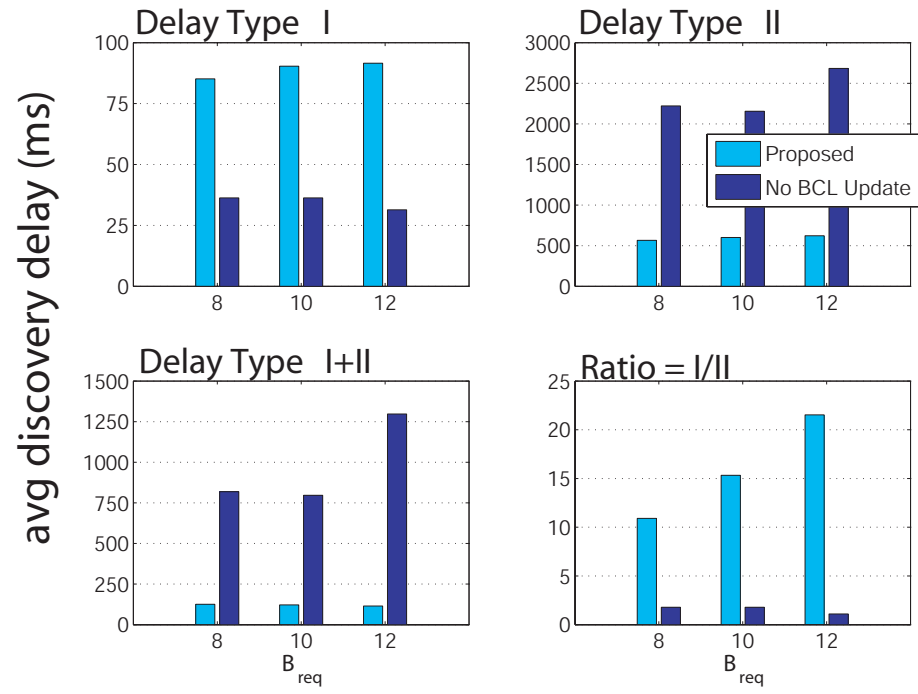

Fig. 9. Test 3: proposed BCL update vs. no BCL update

ways stays with its initial BCL entries. Since estimation plays an important role in updating BCL, both schemes perform the proposed combination of ML and Bayesian estimation.

We set $N=20$ to have enough candidate channels after the initial BCL construction, and vary $B_{\text {req }}$ to be 8 , 10, 12. The BCL update parameters are set as follows: $t U P D A T E=3, t N O \_I M P O R T \_T I M E R=3$, thPOTENTIAL $=$ 0.9, thPOTENTIAL $L_{\text {lower }}=0.88$, thPOTENTIAL $L^{\text {upper }}=0.93$, $N_{\text {lower }}=5$, and $N^{\text {upper }}=15$. Other simulation parameters are listed in Table III.

Fig. 9 plots the simulation results. Both schemes start with the same set of BCL entries where $N^{*}=7$ initially. At the first look, one might think that the proposed scheme performs more poorly than the latter scheme, based on the results on Type-I delay. However, we should not overlook the fact that the latter scheme fixes the size of BCL at 7. Therefore, its TypeI delay never exceeds the sum of sensing-times of the seven channels. On the other hand, our scheme flexibly adjusts its BCL size from 5 to 15 , to maximize the chance of successful opportunity discovery at the first round of searching backup channels. Naturally, the Type-I delay of our scheme could be longer than that of the no-update scheme.

Then, what would be the benefit of adjusting the size/entries of BCL by sacrificing the Type-I delay? To present the benefits clearly, we have counted the number of events when 


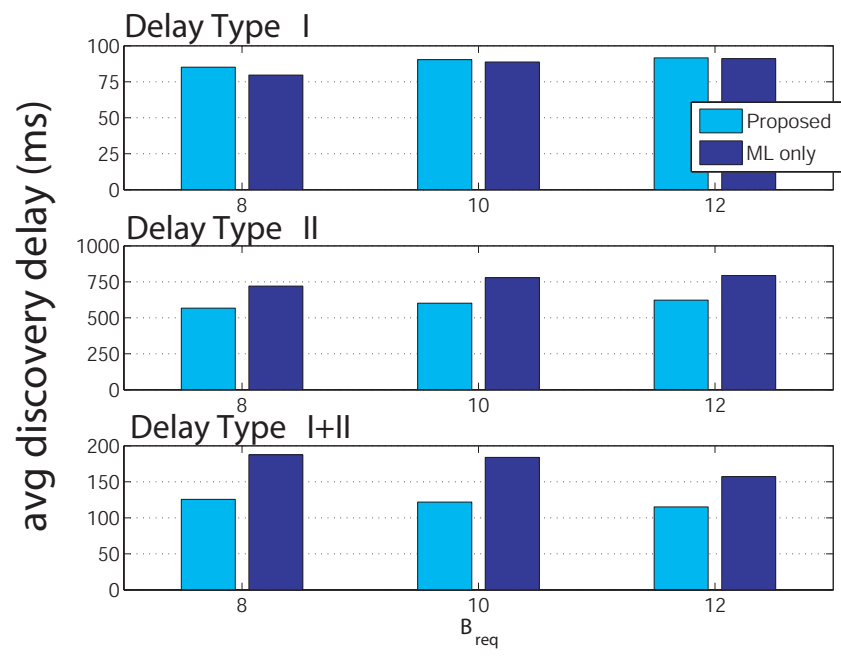

Fig. 10. Test 4: proposed ML/Bayesian combined vs. ML-only strategy

opportunity discovery completes without retries (Type-I) and after retries (Type-II). We calculated the ratio between two events (Type-I / Type-II) and plotted it at the bottom right corner of the figure. It is clear that our scheme incurs TypeI event a lot more than Type-II, which helps reduce the overall opportunity discovery-delay (Type I+II) significantly because Type-II retries are very costly. As a result, our scheme outperforms the no-update scheme in both Type-II delay and the overall delay (I+II), showing up to $76.78 \%$ and $91.12 \%$ of performance enhancement.

\section{Test 4: Proposed ML/Bayesian Combined Estimation Strat- egy vs. ML Estimation Only}

In Test 4, performance of the proposed combination of ML and Bayesian estimation is compared with a ML-only scheme. Both schemes adopt the proposed sensing-sequence and BCL update algorithm. MAX_BS_STAGE is set to 4, where it may be increased to have better performance (i.e., more accurate estimates) at the expense of computational complexity. Other simulation parameters are the same as Test 3 .

As shown in Fig. 10, the proposed strategy achieves a smaller average delay than ML-only estimation by enhancing Type-II delay by up to $22.72 \%$. As a result, the overall delay (I+II) is enhanced by up to $33.75 \%$ over the ML-only strategy. This is because Bayesian inference on class-S channels provides more accurate estimates than ML estimation, which helps update BCL more efficiently.

\section{CONCLUSIONS}

In this paper, we proposed a (sub)optimal sensing-sequence for fast discovery of spectrum opportunities so that a CRN can provide a seamless service to its SUs with minimal QoS degradation. We also proposed construction of a backup channel list (BCL) with an efficient BCL update algorithm to support fast opportunity discovery. Finally, we introduced a combined estimation strategy with ML and Bayesian inference to provide reliable estimation of ON/OFF channel-usage patterns and accurate prediction of channel availability with infrequent and limited samples.
In future, we would like to enhance the proposed suboptimal algorithm so that it can achieve near-optimal performance under various channel conditions.

\section{ACKNOWLEDGMENTS}

The work reported in this paper was supported in part by the National Science Foundation under grants CNS 0519498 and 0721529 , and by Intel Corporation.

\section{REFERENCES}

[1] C.-T. Chou, S. Shankar N, H. Kim, and K. G. Shin. What and how much to gain by spectral agility. IEEE Journal on Selected Areas in Communications, 25(3):576-588, April 2007.

[2] H. Kim and K. G. Shin. Efficient discovery of spectrum opportunities with MAC-layer sensing in cognitive radio networks. IEEE Transactions on Mobile Computing, 7(5):533-545, May 2008.

[3] IEEE 802.22 working group on wireless regional area networks. http://www.ieee802.org/22/.

[4] N.B. Chang and M. Liu. Optimal channel probing and transmission scheduling for opportunistic spectrum access. In Proc. of ACM MobiCom, pages 27-38, September 2007.

[5] N.B. Chang and M. Liu. Competitive analysis of opportunistic spectrum access strategies. In IEEE INFOCOM, pages 1535-1542, April 2008.

[6] Q. Zhao, L. Tong, A. Swami, and Y. Chen. Decentralized cognitive MAC for opportunistic spectrum access in ad hoc networks: A POMDP framework. IEEE JSAC, 25(3):589-600, April 2007.

[7] A. Motamedi and A. Bahai. MAC protocol design for spectrum-agile wireless networks: Stochastic control approach. In Proc. of the IEEE DySPAN 2007, pages 448-451, April 2007.

[8] J. O. Berger. Statistical Decision Theory and Bayesian Analysis, second edition. Springer Science, New York, NY, 2006.

[9] D. Datla, R. Rajbanshi, A.M. Wyglinski, and G.J. Minden. Parametric adaptive spectrum sensing framework for dynamic spectrum access networks. In Proc. of IEEE DySPAN, pages 482-485, Apr. 2007.

[10] C.R. Stevenson, C. Cordeiro, E. Sofer, and G. Chouinard. Functional requirements for the 802.22 WRAN standard. IEEE 802.22-05/0007r47, January 2006.

[11] N. Nie and C. Comaniciu. Adaptive channel allocation spectrum etiquette for cognitive radio networks. Mobile Networks and Applications (MONET), 11(6):779-797, December 2006.

[12] R. Rajbanshi, Q. Chen, A. M. Wyglinski, G. J. Minden, and J. B. Evans. Quantitative comparison of agile modulation techniques for cognitive radio transceivers. In the First IEEE Workshop on Cognitive Radio Networks (IEEE CCNC 2007), pages 1144-1148, January 2007.

[13] H. Kim, C. Cordeiro, K. Challapali, and K. G. Shin. An experimental approach to spectrum sensing in cognitive radio networks with off-theshelf IEEE 802.11 devices. In the First IEEE Workshop on Cognitive Radio Networks (IEEE CCNC 2007), pages 1154-1158, January 2007.

[14] S. Geirhofer, L. Tong, and B. M. Sadler. Dynamic spectrum access in the time domain: Modeling and exploiting white space. IEEE Communications Magazine, 45(5):66-72, May 2007.

[15] D. R. Cox. Renewal Theory. Butler \& Tanner Ltd, London, UK, 1967.

[16] C. Cordeiro, K. Challapali, and M. Ghosh. Cognitive PHY and MAC layers for dynamic spectrum access and sharing of TV bands. In Proc. of the ACM TAPAS 2006, August 2006.

[17] A. Ghasemi and E. S. Sousa. Collaborative spectrum sensing for opportunistic access in fading environments. In Proc. of the IEEE DySPAN 2005, pages 131-136, November 2005.

[18] G. Ganesan and Y. Li. Cooperative spectrum sensing in cognitive radio networks. In IEEE DySPAN 2005, pages 137-143, November 2005.

[19] S. M. Mishra, A. Sahai, and R. W. Brodersen. Cooperative sensing among cognitive radios. In Proc. of the IEEE ICC 2006, pages 16581663, June 2006.

[20] T. Clancy. Formalizing the interference temperature model. Wiley Journal on Wireless Communications and Mobile Computing, 7(9):10771086, November 2007.

[21] C. Cordeiro, K. Challapali, D. Birru, and S. Shankar N. IEEE 802.22: An introduction to the first wireless standard based on cognitive radios. Journal of Communications (JCM), 1(1):38-47, April 2006.

[22] D. Simon. Optimal State Estimation: Kalman, $H_{\infty}$, and Nonlinear Approaches. John Wiley \& Sons, Inc., Hoboken, NJ, 2006. 